\title{
Forward Physics Measurements at the LHC
}

\author{
Risto Orava ${ }^{1}$ \\ Helsinki Institute of Physics and University of Helsinki \\ P.O. Box 64, 00014 University of Helsinki, Finland \\ E-mail: risto.orava@helsinki.fi
}

\begin{abstract}
A unique experiment is under making at CERN. Forward physics practitioners of the TOTEM, CMS and FP420 collaborations are combining their efforts to set up a spectrometer that covers more phase space than any other collider experiment so far. The aim is to measure properties of the strong interaction in extreme conditions. The experiment will also search for novel physics phenomena by using a highly selective process facilitated by colour singlet exchange: $p p \rightarrow p+$ $X+p$ and characterized by a forward-backward pair of leading protons and a central system with the quantum numbers $J^{P C}=0^{++}$.

The forward physics program is highly complementary to the base line LHC physics scenarios: elastic and diffractive scattering or low- $x$ processes could not be investigated without further tracking and calorimetry at small polar angles. With the extended detector coverage, physics runs with different optics choices will allow the LHC to be used to its full - and effectively transform the machine into a laboratory of QCD asymptotics (elastic and diffractive scattering), deeply inelastic scattering (low- $x$ processes), and gluon physics (central exclusive diffraction).
\end{abstract}

International Workshop on Diffraction in High-Energy Physics-Diffraction 06Adamantas, Milos island, Greece 5-10 September 2006 


\section{Introduction}

In order to maximally cover the signatures of forward physics processes - leading forward protons and clusters of particles separated by gaps in rapidity - the base line CMS experiment is extended (see Fig. 1) by (1) the TOTEM Roman Pots at $\pm 147 \mathrm{~m}$ and $\pm 220 \mathrm{~m}$ locations, (2) TOTEM T1 and T2 track stations at \pm 10 and $\pm 14 \mathrm{~m}$, and by the (3) CMS CASTOR and Zero Degree (ZDC) calorimeters at \pm 20 and $\pm 170 \mathrm{~m}$ on both sides of the interaction point number 5 (IP5), [1]. In addition, it is foreseen that further leading proton detectors will be added to \pm 420 meters for covering, with the nominal LHC optics, the medium central diffractive masses of the order of ${ }^{1} 60-250 \mathrm{GeV}$.

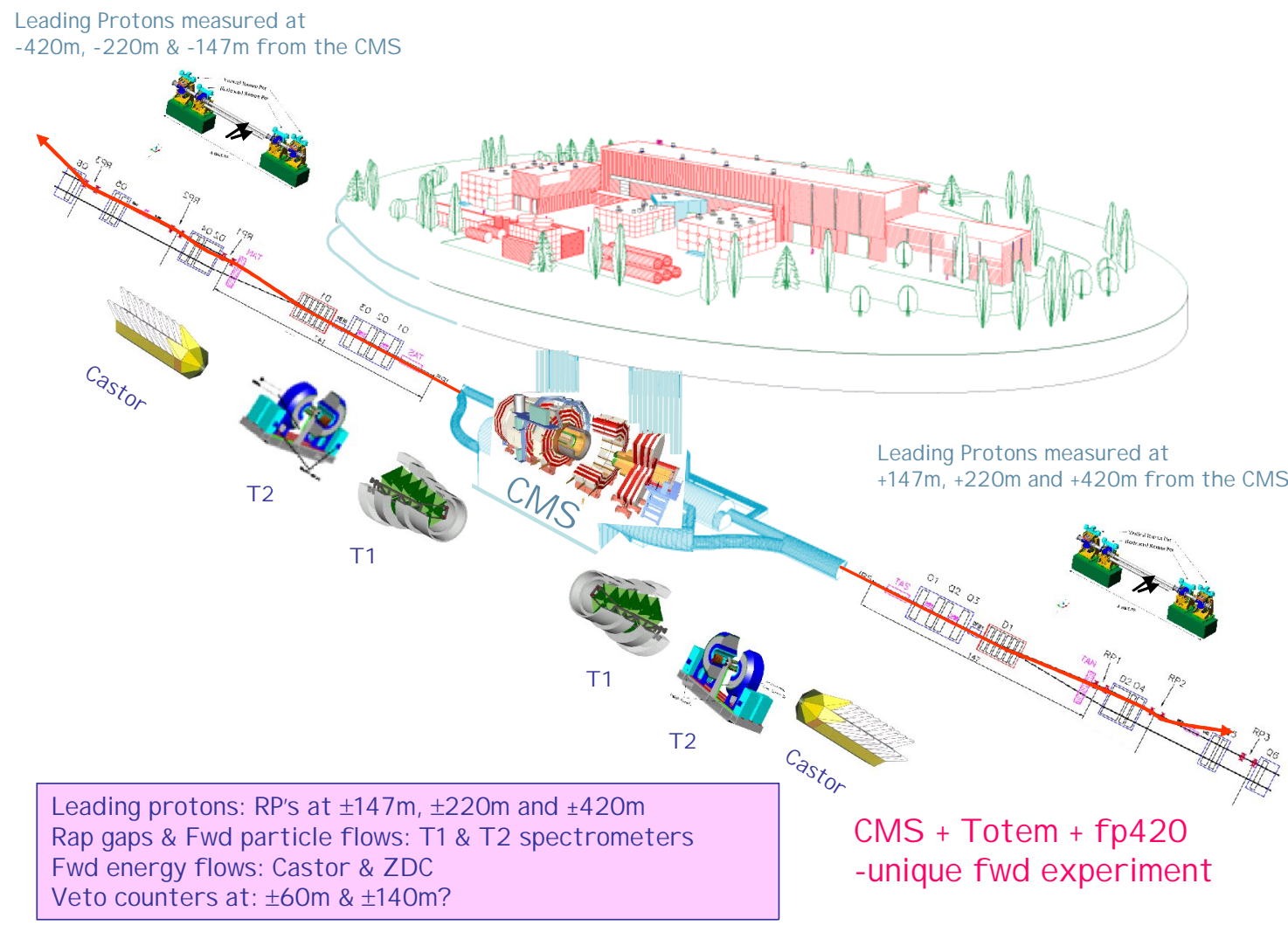

Figure 1 Lay-out of the TOTEM and CMS detectors to be used in measuring forward physics processes at the LHC.

The geometric acceptance of the forward spectrometer is depicted in Fig. 2 for transverse momenta $\left(p_{\mathrm{T}}\right)$ vs. pseudorapidity $(\eta)$ of the final state particles. For reference, the tracking acceptances of the four LHC experiments are also shown.

\footnotetext{
${ }^{1}$ For the fp420 R\&D proposal see: http://www.fp420.com/papers/fp420_UK_2006.pdf
} 


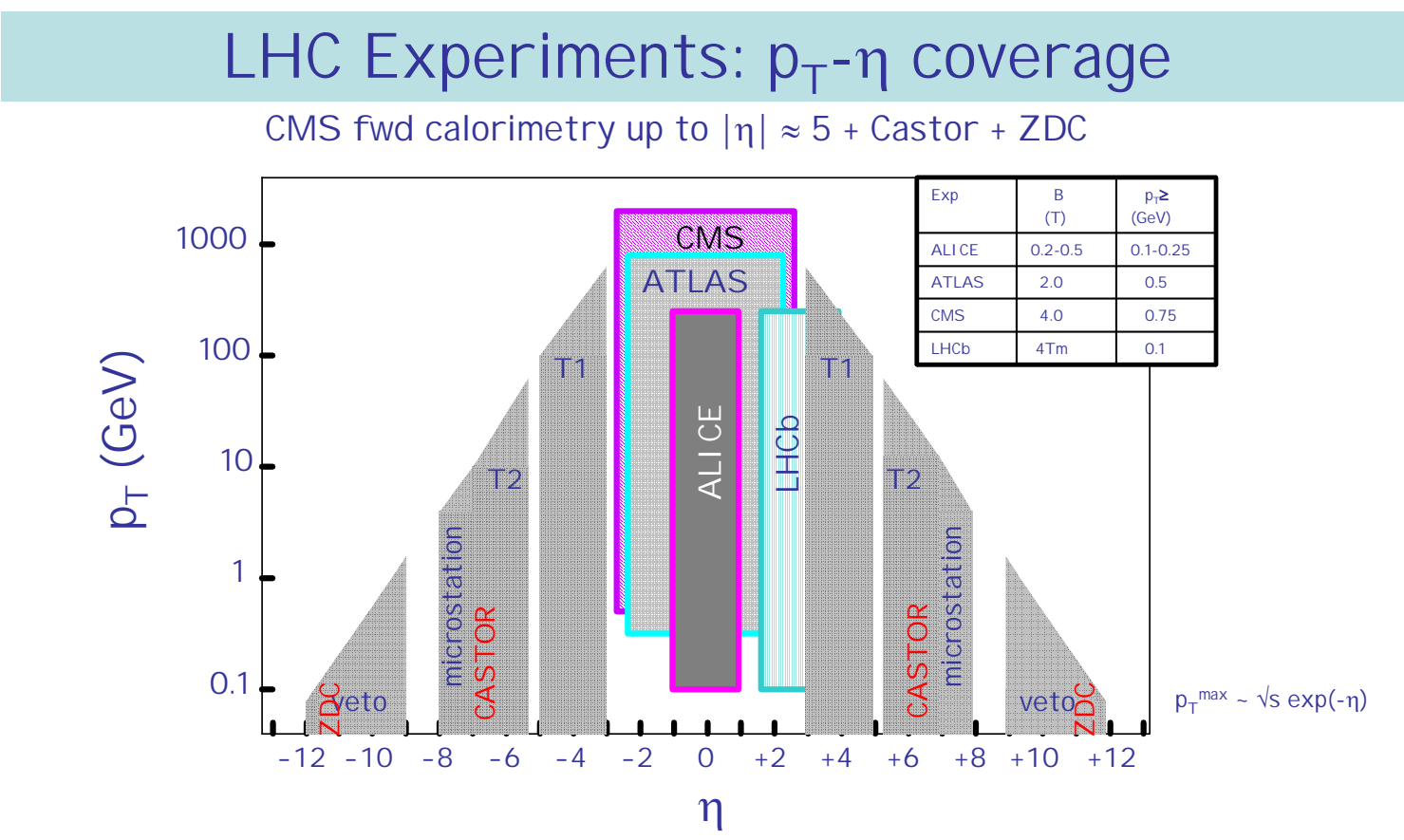
The base line LHC experiments will cover the central rapidity region. TOTEM円CMS + will complement the coverage in the forward region.$$
+\mathrm{fp} 420 \mathrm{~m}
$$
R. Orava Diffraction 2006 Milos Island

Figure 2 Geometric acceptance of the TOTEM and CMS detectors to be used in measuring forward physics processes at the LHC. Acceptances of the tracking detectors of ALICE, ATLAS, CMS and LHC-b experiments are shown as a reference.

The TOTEM experiment aims at measuring (1) the total proton-proton cross section with a relative precision of $\approx 1 \%$, (2) elastic proton-proton scattering for the four-momentum squared, $-t$, between $10^{-3}<-t \approx(p \theta)^{2}<10 \mathrm{GeV}^{2}$. In addition, TOTEM will provide precise measurements of the leading forward protons with longitudinal momentum losses, $\xi$, from $2 \times 10^{-3}$ and particle flows and rapidity gaps in the pseudorapidity regions of $3.1<\eta<4.7$ (T1) and $5.3<\eta<6.5$ (T2) for the benefit of studies on soft diffractive scattering. By combining the TOTEM signatures with the CMS and its forward additions, the CASTOR and ZDC calorimeters, a full born forward physics program can be carried out. CASTOR complements the particle flow measurements of TOTEM T2 and ZDC extends the forward measurements to include leading neutral particles at $\theta \approx 0^{\circ}$.

An important gap in the leading forward proton acceptance, for the nominal LHC run conditions, will be filled up by the detectors at \pm 420 meters. These will be crucial additions in searching for a light SM or MSSM Higgs boson in Central Exclusive Diffraction (CED):

$$
p p \rightarrow p+h^{0}+p,
$$

where the plus signs denote rapidity gaps. 


\section{Elastic scattering}

Measurement of the differential elastic cross section, $d \sigma_{e l} / d t$, in the widest possible range in four-momentum transfer, $-t$, will:

- Yield the pp interaction radius, i.e. slope of the $d \sigma_{e l} / d t$ distribution. The unitarity induced corrections first influence the value of the slope parameter.

- Enable, together with the measurement of the total inelastic rate, to detemine the total pp cross section, $\sigma_{\text {tot }}$.

- Test the Coulomb-nuclear interference which is expected to have an effect over large interval in $-t$.

- Yield a measurement of the ratio of the real and imaginary parts of the forward $p p$ scattering amplitude, $\rho=\operatorname{Re} A(s, t) / \operatorname{Im} A(s, t)$. Through dispersion relations, a precise measurement of $\rho$ will constrain $\sigma_{t o t}$ at substantially higher energies.

The elastic proton-proton interactions are measured, and triggered, by the TOTEM leading proton detectors placed symmetrically on both sides of the CMS experiment at ' \pm 147 ' and ' \pm 220 ' meters from the Interaction Point (IP5) ${ }^{2}$. To measure protons at small scattering angles, the detectors must be moved close to the primary LHC beam ${ }^{3}$. The 'nominal' TOTEM beam optics set-up has high $\beta^{*}\left(\beta^{*} \approx 1540 \mathrm{~m}\right)$ and no crossing angle at the IP for optimizing the acceptance and accuracy at small values of the four-momentum transfer squared down to $-t_{\min } \approx 2 \cdot 10^{-3} \mathrm{GeV}^{2}$.

The $-t$ distribution of the scattered protons, $d N_{e l} / d t$, is extrapolated to $-t=0$, where it is related to the total proton-proton cross section by the Optical theorem. With the special optics of $\beta^{*}=90 \mathrm{~m}$, a first (and less precise) measurement of the elastic cross section $d \sigma_{e l} / d t$ could be made; extrapolation to the Optical point will be made with an accuracy of a few percent ${ }^{4}$.

Short special runs with other LHC optics set-ups, such as $\beta^{*}=90 \mathrm{~m}$, the 'injection' optics $\left(\beta^{*}=18 \mathrm{~m}\right)$, or stage-1 'pilot' run optics $\left(\beta^{*}=2 \mathrm{~m}\right)$ will allow measurements up to $-t \approx 10-15$ $\mathrm{GeV}^{2}$. Runs with a reduced center-of-mass energy will allow an analysis of energy dependence, comparisons with the Tevatron results and a precise measurement of the $\rho$-parameter.

\section{Soft diffractive scattering}

Single diffractive dissociation (see Fig. 3), SD, of an initial proton into a final state system with large mass, $M^{*}$, is not straightforward when Regge model is strictly followed and the Pomeron intercept indicated by the experimental measurements of $\sigma_{t o t}$ is used for the predictions. Both

\footnotetext{
${ }^{2}$ The detector locations at \pm 145 and \pm 149 meters from IP5 are here referred as the ' \pm 147 ' meter location and the ones at \pm 216 and \pm 220 meters from IP5 as the ' \pm 220 ' meter location.

${ }^{3}$ The closest approach to the beam is of the order of a few mm's $(10 \sigma+0.5 \mathrm{~mm})$ and depends on the measurement location and used beam optics scheme.

${ }^{4}$ TOTEM Collaboration, TOTEM TDR, CERN-LHCC-2004-002 (2004); The numbers referring to the optics with $\beta^{*}=90 \mathrm{~m}$ are based on preliminary TOTEM studies by J. Kaspar et al., TOTEM Collaboration (2006), concerning the extrapolation to the optical point see also [2].
} 
the triple-Pomeron based description and prediction of "multi-Reggeon" events, i.e. for events with a few large rapidity gaps, lead to a $\sigma^{S D}$ that grows faster than $\sigma_{\text {tot. }}$. A measurement of $\sigma^{S D}$ and the cross section of multiple large rapidity gaps at the LHC will test the proposed models [3].
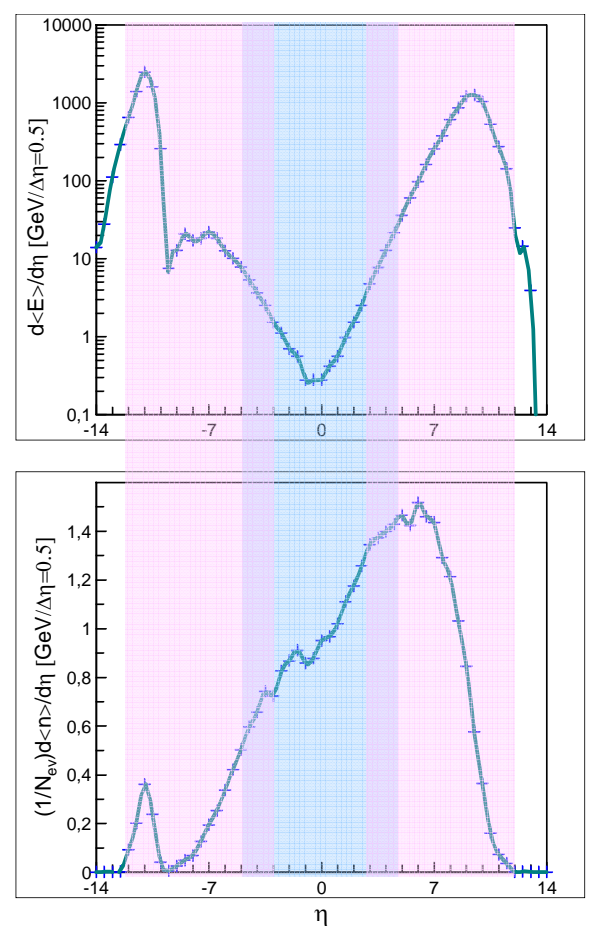

Figure 3 The predicted (PYTHIA) energy flows (upper figure) and multiplicities (lower figure) in an average Single Diffractive (SD) event at the LHC energy (14 TeV). The diffractively scattered leading proton points to left. The shaded regions indicate the acceptances of the CMS experiment (blue) and TOTEM (pink). The small gaps in the baseline TOTEM acceptance are not shown ${ }^{5}$.

For understanding the asymptotics of the strong interaction amplitude, it is crucial to measure the $t$-dependence of $\sigma^{S D}$. Measurement of the cross section $d \sigma^{S D} / d t$ at $t \rightarrow 0$, would put the 'weak coupling' scenario to test.

Measurement of the location of the diffractive minimum, predicted as a result of the destructive interference between the pole and cut contributions, would allow further testing of the 'weak coupling' scheme. It will be especially important to measure the $t$-dependence for exclusive channels as the spin-flip amplitudes as well as varying strengths of the rescatterings for different masses leads cancellation of the diffractive minimum.

A variety of models for controlling the growth of $\sigma^{S D}$ has been suggested in the literature. In the $s$-channel picture, blackening of the interactions at small impact parameters strongly suppresses inelastic diffraction and in Regge calculus, the multi-Pomeron 'screening effects' should be larger for SD than for the elastic scattering, i.e. the gap survival factor $S^{2} \rightarrow 0$ when c.m.s. energy, $s \rightarrow \infty$, or the gap size $\Delta \eta \rightarrow \infty$. It is important to investigate the dependence of $S^{2}$ on c.m.s. energy, gap size and the number of gaps.

\footnotetext{
${ }^{5}$ Simulation by Jerry Lämsä (2006).
} 
Since SD is supposedly dominated by the periphery of the interaction disk, characteristics of the particles produced within the diffractive system could be distinguishable by, e.g. their smaller average transverse momenta and different dispersion of the hadron multiplicity distribution as compared to the inclusively produced secondaries at energies $\sqrt{s}=M_{X}$. Also long range rapidity correlations may be induced between the final state hadrons in single diffractive processes. It is important to test these experimentally.

Measurement of soft diffractive processes is based on tagging leading protons and rapidity gaps - beyond statistical (exponential) multiplicity fluctuations - in inelastic events. The cross sections are typically large, and short special runs with the 'nominal' TOTEM high $\beta^{*}$ optics conditions $\left(\beta^{*} \approx 1540 \mathrm{~m}\right)$ will yield excellent statistics for these types of events. The pilot runs with 'custom' optics such as $\beta^{*}=90 \mathrm{~m}$, planned for the initial stages of the LHC operation, are well suited for measurements of soft diffractive scattering.

TOTEM registers about $85 \%(50 \%)$ of the diffractive protons, over most of the $\xi-t$ plane with the nominal $\beta^{*}=1540 \mathrm{~m}$ (custom $\beta^{*}=90 \mathrm{~m}$ ) and has a diffractive mass, $M^{*}$, acceptance better than $50 \%$ down to $M^{*}=3 \mathrm{GeV}$. With the nominal LHC optics $\left(\beta^{*}=0.55 \mathrm{~m}\right)$, diffractive protons with $\xi \geq 0.02$ will be seen at the $\pm 220 \mathrm{~m}$ location and with $0.002 \leq \xi \leq 0.018$ at the \pm 420 m location [4].

\section{Semi-hard diffractive scattering}

The semihard diffractive processes are characterized by soft diffractive dynamics combined with the objects signifying the hard scale within the diffractive system, i.e. high ET jets, heavy quarks, heavy bosons and possible new heavy particles. By measuring these objects up to rapidities of $|\eta| \approx 6.5$, the quark and gluon structure of diffraction ${ }^{6}$ can be examined by evaluating the Bjorken- $\mathrm{x}, x_{B j}$, as:

$$
x_{B j} \equiv \frac{1}{\sqrt{S}} \sum_{i=1}^{n} E_{T}{ }^{i} e^{-\eta^{i}}
$$

where $E_{T}^{i}$ is the transverse energy of object $i$ (a jet or another heavy object $i$ within the diffractive system) and $\eta_{i}$ its pseudorapidity. The ratio $\beta \equiv x_{B j} / \xi$ could then be interpreted as the momentum fraction of a parton within the Pomeron.

In the Pomeron based model, the cross sections of the above 'hard' subprocesses can be calculated by convoluting the initial state parton distributions in the proton and in Pomeron with the cross section of the 'hard' subprocess in question. The expected cross sections are large, of the order of 1-10 nb. The measurements of these subprocesses at the LHC will put this theoretical model to test.

The kinematics of these events is defined by the invariant mass and pseudo-rapidity of the diffractive system. The measurement aims at reconstructing the produced hard objects within the full acceptance region of the TOTEM $\oplus$ CMS set-up.

\footnotetext{
${ }^{6}$ This is supposed to measure the 'Pomeron' structure, using the Regge language. However, it is known that in low impact parameter reactions a single Pomeron does not dominate diffraction.
} 
Semihard diffractive events will be recorded during the runs with nominal TOTEM and custom optics of $\beta^{*} \cong 1540 \mathrm{~m}, 90 \mathrm{~m}$ and $18 \mathrm{~m}$ and during the nominal CMS $\oplus$ TOTEM LHC runs for the pseudorapidity range of $3.1<|\eta|<6.5$ (CASTOR and T2 spectrometer) allowing the Bjorken-x to be measured down to about $x_{B j}=10^{-6}-10^{-7}$.

\section{Low- $x$ physics}

The detection of low-x processes is based on tagging the objects (jets, heavy quarks, heavy bosons) that signify a hard scale in both central and forward rapidities.

TOTEM $\oplus$ CMS could test a number of specific predictions based on the optical low- $x$ model by Frankfurt, Strikman and Weiss [5], by measuring:

- The type of leading particles, in particular nucleons and their resonant $\left(\mathrm{N}^{*}\right)$ states.

- Transverse momenta of the leading particles for $p_{\perp} \geq 1 \mathrm{GeV} / \mathrm{c}$ and correlations between the transverse momenta of leading hadrons ${ }^{7}$.

- Particle flows for particles in the fractional momentum region of $z \geq 0.02-0.05$ in both fragmentation regions (long range rapidity correlations) and particle/energy flows at (pseudo)rapidities of $\eta=4-6$.

The aim of the TOTEM $\oplus$ CMS set-up is to measure jets at rapidities $\eta \approx 6.5$ up to transverse energies of $E_{T} \approx 10 \mathrm{GeV}$. This allows $x$-values of $x \approx 10^{-6}$ to be reached and effectively transforms LHC into a deeply inelastic scattering (DIS) machine. Correlations between the forward jets, generated mostly through interactions with partons at very small $x\left(x<<10^{-2}\right)$ and central jets $\left(x>10^{-2}\right)$, heavy quarks and heavy bosons yield detailed information of the proton parton configurations in three dimensions. The forward jets are also proposed to be used in triggering for new phenomena in high- $E_{T}$ central processes.

The CASTOR calorimeter would cover the pseudorapidity region of $5.4<\eta<6.7$, similar to the coverage of the $\mathrm{T} 2$ tracking station, and would be of help in measuring the forward jets. With a modest detector upgrade [6], and with the proposal recently presented in [3.1], the rapidity acceptance could be extended up to $|\eta| \approx 11$ allowing Bjorken- $x$ values down to $x \sim$ $10^{-8}$ to be measured.

\section{Central Diffraction}

\subsection{Soft Central Diffraction}

A good environment for the production of $c$-meson states, such as $\chi_{c}$, via $g g \rightarrow M$ is expected [7] in the CED process

$$
p p \rightarrow p+\chi_{c}+p
$$

A strong coupling could be expected for glueballs ${ }^{8}$ (or supersymmetric gluinoballs) and hybrids as a result of the assumed two-gluon exchange. The very high statistics studies possible with the 'gluon collider' mode of operation (3) at the LHC will provide this possibility. In this

\footnotetext{
${ }^{7}$ The acceptances to be verified experimentally.

${ }^{8}$ The $\beta^{*}=90 \mathrm{~m}$ optics may not provide adequate mass resolution for the low-mass states.
} 
case, azimuthal correlations with the quasi-elastic pair of leading protons can be particularly significant [8].

The measurement of Soft Central Exclusive Diffractive (CED) interactions requires tagging the leading diffractive protons (or their diffractive excitations) and/or rapidity gaps between the produced systems and the central system. Identification and measurement of central activity, leptons, $c$ - and $b$-quarks increases the physics potential of the LHC and helps in rejecting QCD backgrounds. Forward coverage is important in filtering out the contamination expected from the inclusive central diffractive (CD) processes.

In case of low mass $\mathrm{CD}$ interactions, relatively short runs with the special machine optics with $\beta^{*}=90 \mathrm{~m}$ are used.

The absence of large $p_{\perp}$ presents a challenge for triggering on these final states; low multiplicity and the presence of the rapidity gaps and the pair of leading protons should help in tagging the events.

\subsection{Hard Central Diffraction}

Measurements of the Central Exclusive Diffractive (CED) processes of the type [9]

$$
p p \rightarrow p+M_{X}+p
$$

will significantly extend the physics programme at the LHC [10]. The process facilitates both QCD asymptotics and searches for physics beyond the standard model. Theoretically, hadronic processes containing large rapidity gaps play a key role in determining the asymptotic behaviour of the cross sections at high energies. Experimentally, the presence of rapidity gaps provides a clean environment for investigating the signal events. In such events, a colour singlet state, $M_{X}$, is produced with a minimal background from soft secondary particles. Process (3) effectively turns the LHC $p p$ collider into a gluon factory [6].

In case of low mass $C D$ interactions, relatively short runs with the special machine optics with $\beta^{*}=90 \mathrm{~m}$ are used. In searching for the CED Higgs events with central masses below 250 $\mathrm{GeV}$, the leading proton measurement stations at $\pm 420 \mathrm{~m}$ from the IP and the nominal LHC optics with $\beta^{*}=0.55 \mathrm{~m}$ is required. For central masses in excess of $250 \mathrm{GeV}$, low- $\beta^{*}$ conditions together with the leading proton measurement at the $\pm 220 \mathrm{~m}$ location are needed (see Fig. 4).

The cross section for the CED process (Fig. 5): $p p \rightarrow p+H+p$ where the + signs indicate rapidity gaps, can be calculated as the convolution of the effective diffractive luminosity $L\left(\mathrm{gg}^{\mathbb{P P}}\right)$, and the square of the matrix element of the corresponding subprocess [11]. The cross section is predicted to be small [12]: $\sigma_{\text {excl }}(H) \sim 10^{-4} \sigma_{\text {incl }}^{\text {tot }}(H)$. 

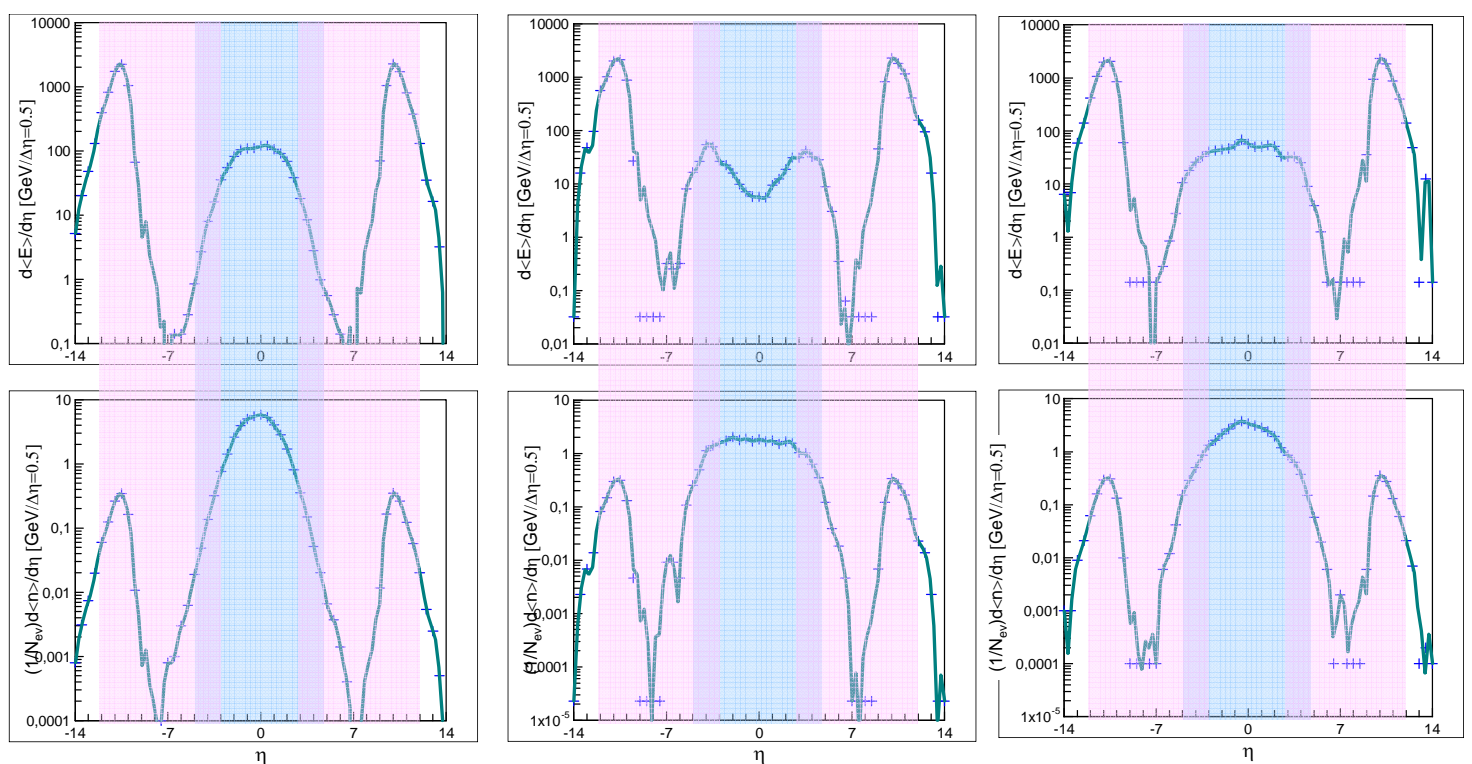

Figure 4 The predicted (PHOJET) energy flows (upper panels) and multiplicities (lower panles) in an average Central Inclusive Diffractive (CID) events at the LHC energy (14 TeV). The pairs of leading protons are selected to be within the acceptance of the leading proton detectors; (1) (left panels): $\pm 220 \mathrm{~m}$ location $(0.02<\xi<0.18)$ for the low- $\beta^{*}$ runs $\left(\beta^{*}=0.55,2\right.$ or $\left.18 \mathrm{~m}\right)$, yielding central mass acceptance of $M_{X}>280 \mathrm{GeV}$; The efficiency for selecting these events is 15\%, (2) (middle panels): $\pm 420 \mathrm{~m}$ location $(0.0022<\xi<0.17$ for clockwise and $0.0018<\xi<0.13$ for counter-clockwise protons $)$ for low- $\beta^{*}$ runs $\left(\beta^{*}=0.55 \mathrm{~m}\right)$, yielding the central mass acceptance of $50<M_{X}<150 \mathrm{GeV}$; The efficiency for selecting these events is $21 \%$, (3) (right panels): \pm 220 m location $(0.024<\xi<0.20$ for clockwise and $0.026<\xi<$ 0.20 for counter-clockwise protons $)$ and at the $\pm 420 \mathrm{~m}$ location $(0.0022<\xi<0.017$ for clockwise and $0.0018<\xi<0.013$ for counter-clockwise protons $)$ for low- $\beta^{*}$ runs $\left(\beta^{*}=0.55 \mathrm{~m}\right)$, yielding the central mass acceptance of $50<M_{X}<800 \mathrm{GeV}$; The efficiency for selecting these events is $37 \%$. The shaded regions indicate the acceptances of the CMS experiment (blue) and TOTEM (pink). The small gaps in the baseline TOTEM acceptance are not shown ${ }^{9}$.

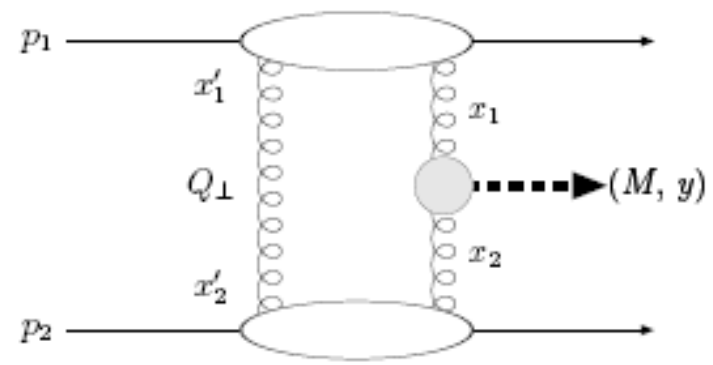

Figure 5 The two-gluon exchange process used in calculations in [11].

The exclusive reaction has several advantages compared to the inclusive ones:

\footnotetext{
${ }^{9}$ Simulation by Jerry Lämsä (2006).
} 
- The Higgs mass (and maybe its width) can be measured with a high accuracy of the order of $\sigma(M)=1 \mathrm{GeV}$ by using the forward-backward pair of leading protons [8].

- The leading order $\mathrm{b} \overline{\mathrm{b}} \mathrm{QCD}$ background is suppressed by the $P$-even $J_{z}=0$ selection rule [6] where the $z$-axis is along the direction of the proton beam. Therefore one can observe the Higgs boson via the main decay mode $\mathrm{H} \rightarrow \mathrm{b} \overline{\mathrm{b}}$. Moreover, a measurement of the mass of the decay products must match the 'missing mass' measurement. It should be possible to achieve a signal-to-background ratio of the order of ${ }^{10} 1$.

- The quantum numbers of the central object (in particular, the $C$ - and $P$-parities) can be analysed by studying the azimuthal angle distribution of the tagged leading protons [13].

- The exclusive CDE events are experimentally clean since the soft background is strongly suppressed.

- Extending the study to SUSY Higgs bosons, there are regions of SUSY parameter space where the signal is enhanced by a factor of 10 or more, while the background remains unaltered. There are regions where the conventional Higgs signals are suppressed and the CED signal is enhanced, even such that the $h$ and $\mathrm{HO}^{++}$bosons may be detected [14].

The results of the first systematic analysis of the precision of the measurement of the leading proton momentum and the accuracy of the reconstructed mass are reviewed in [8]. The analysis is done by tracking the scattered protons along the LHC beam line using the nominal $\beta^{*}=0.55$ $\mathrm{m}$ LHC optics, accounting for uncertainties related to beam transport, and the detection of the scattered protons.
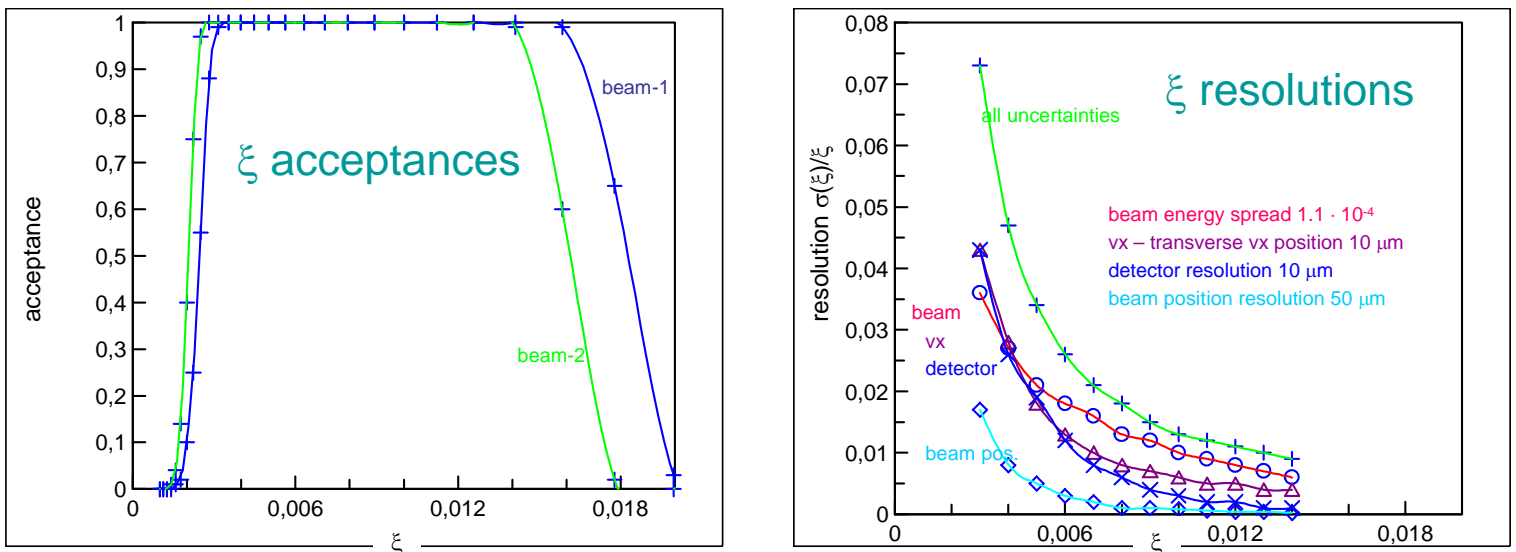

Figure $6 \xi$-acceptances of leading diffractive protons measured at the $\pm 420 \mathrm{~m}$ location in reaction (3) for the protons advancing to clockwise (beam-1) and anti-clockwise (beam-2) directions along the LHC ring (left panel). Resolution is $\xi$ of the clockwise (beam-1) protons detected at the $\pm 420 \mathrm{~m}$ location (right panel) [4].

The relative resolution on $\xi, \Delta \xi / \xi=\left(\xi-\xi_{\text {rec }}\right) / \xi$ as a function of $\xi$ for protons circulating in the clockwise and counter-clockwise directions is shown in Fig. 6 for the $\pm 420 \mathrm{~m}$ location. Included are the separate effects from the uncertainty of the transverse IP position, the

\footnotetext{
${ }^{10}$ The role of the pile-up and machine related backgrounds is being actively worked on (see, for example: Andy Pilkington, FP420 Collaboration and Marek Tsasevsky, Ref [1]).
} 
resolution of the proton detector, the beam energy uncertainty, the beam angular divergence at the IP, and the beam position resolution at the proton detector.

To search for and precisely measure new particle states with masses below $200 \mathrm{GeV}$, the additional leading proton detectors at $\pm 420 \mathrm{~m}$ on both sides of IP5 are needed in addition to the already approved detectors. Using these, a mass resolution of the order of $1 \mathrm{GeV}$ can be achieved for masses beyond $\sim 100 \mathrm{GeV}$ (Figs. 7 and 8).
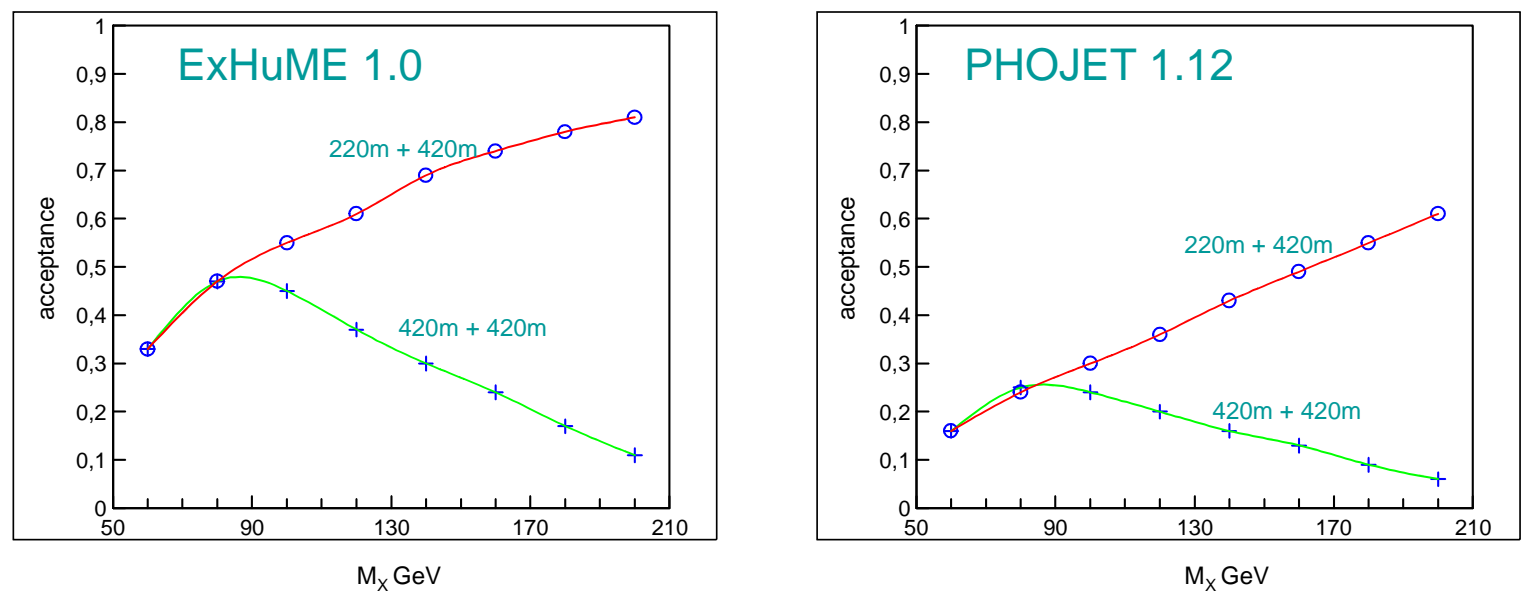

Figure 7 Mass acceptance for the central masses, $M_{X}$, in $p p \rightarrow p+M_{X}+p$ as simulated by using the ExHuME and PHOJET Monte Carlo models [4].

The acceptance as a function of the mass of the centrally produced system is shown in Fig. 7. Each leading proton is required to be within the acceptance of either the \pm 215 or $\pm 420 \mathrm{~m}$ location. Independently shown is the case (subset of the above) where both protons are within the acceptance of the $\pm 420 \mathrm{~m}$ locations. In the mass range shown, there is no acceptance for detecting both protons at the $\pm 215 \mathrm{~m}$ location. The event generators ExHuME and PHOJET were used for the simulations.

The $\xi_{1}-\xi_{2}$ combinations result from the initial state gluon density functions in the proton and the mass of the centrally produced system. The ExHuME generator [15], which is based on the perturbative QCD calculation of [16], favours a harder gluon distribution than that employed in PHOJET and thus the produced Higgs bosons are more central in rapidity. This yields a higher acceptance for ExHuME than for PHOJET for the same mass.

The resolution effects of the two scattered protons are, in general, uncorrelated from each other. The only correlation comes from the production point, whose transverse component is determined by the r.m.s. spread of the beam at the IP and by an independent measurement using the Higgs decay products [4].

The mass resolutions for events with protons within the acceptance of the \pm 420 m location on both sides, and for events with one proton within the acceptance of the $\pm 215 \mathrm{~m}$ location on one side and the other proton within the acceptance of the $\pm 420 \mathrm{~m}$ location on the other side are shown as a function of the mass of the centrally produced system in Fig. 8. The values quoted in the figure are based on Gaussian fits to the reconstructed mass distributions. The two-proton 
acceptance requirement imposes a restriction on the allowed $\xi_{1}-\xi_{2}$ combinations; as a result the mass resolutions obtained with ExHuME and PHOJET are very similar.

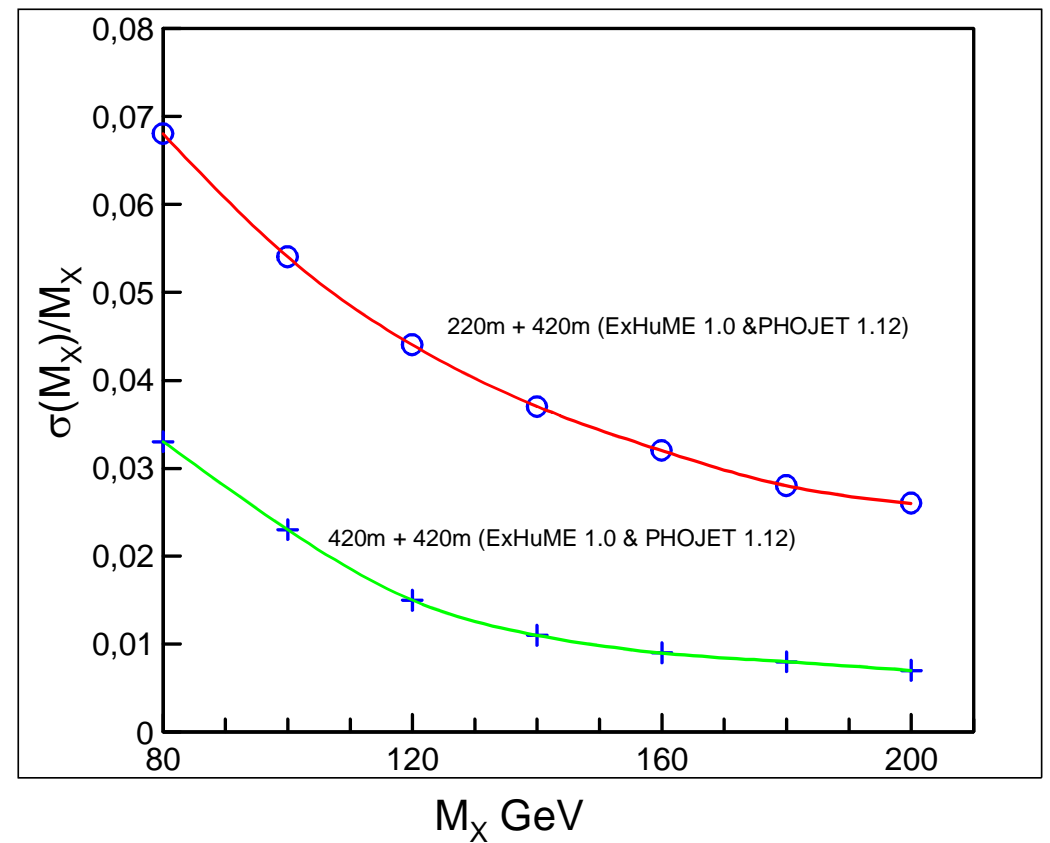

Figure 8 Mass resolution expected for the central masses, $M_{X}$, reconstructed in $p p \rightarrow p+M_{X}+p$ by measuring the leading diffractive protons at the $\pm 420 \mathrm{~m}$ and $\pm 220 \mathrm{~m}$ locations. The lower curve assumes that both diffractive protons are measured at the $\pm 420 \mathrm{~m}$ leading proton detectors; the upper curve is based on measuring one of the leading protons at the $\pm 420 \mathrm{~m}$ and another one at the $\pm 220 \mathrm{~m}$ location [4].

\section{Summary}

The physics scenario of TOTEM is based on (1) short special high statistics runs which begin during the running-in stages of the machine, and (2) forward physics runs in conjunction with the CMS experiment with the nominal low- $\beta^{*}$ machine conditions. The focus is on physics that is complementary to the general purpose experiments at the LHC. The base line TOTEM experiment comprises Roman Pot detectors for the leading proton measurement, and the two tracking stations (T1 and T2); the forward region of IP5 is further extended by the CMS CASTOR and ZDC calorimeters.

The analysis foresees later stage low- $\beta^{*}$ runs, and also the well-known Central Exclusive Diffractive interaction: $p p \rightarrow p+H+p$ which is considered as a benchmark process, with an exclusive access to the $J^{P C}$ structure of the Higgs boson.

The physics program of the experiment consists of the following:

1. Elastic proton-proton scattering, total proton-proton cross section, soft and hard diffraction. The proton structure can be investigated beyond the usual longitudinal parton density functions, and pure gluon jet analyses with large statistics will become available. The T2 tracker enables proton gluon densities to be measured down to a Bjorken- $x$ of $10^{-6}$, the 
dynamics of rapidity gap survival (including the phenomena behind the generation of multiple gaps) can be extended to rapidities of close to 7 units $^{11}$. Physics of the proton light cone could be studied in kinematical configurations that have not been available to any previous experiment. In addition, more detailed features of diffractive and small- $x$ processes, such as jet, $W, J / \psi, b$ - and $t$-quark production, will be available to experimentation. Possible saturation effects of the parton densities, BFKL dynamics and multi-parton scattering could be investigated by the TOTEM experiment.

2. With the leading proton tagging (with a possible later stage extension to the 420 meter region) and the $\mathrm{T} 1$ and $\mathrm{T} 2$ spectrometers, a threshold scan for spin-parity selected new particle states, including the Higgs boson, SUSY particle states, radions, extra dimensions etc. will be available.

3. The forward detectors of TOTEM and CMS provide an extension to the kinematic reach of CMS, and enable luminosity independent measurement and monitoring of the LHC luminosity. With its excellent forward acceptance, TOTEM could be of crucial help in studying the minimum bias events and in jet energy calibration of the CMS calorimeters.

The forward physics road map relies on tagging the leading protons, identifying the rapidity gaps within a sufficiently large $\eta$ range, and by rejecting the backgrounds by track and doublearm coincidence selection by the Roman Pots and T1 \& T2 detector stations. In addition to the physics reach discussed here, Drell-Yan processes, photon-proton, photon-photon and other forward physics processes are being studied [1].

\section{References}

Prospects for diffractive and forward physics at the LHC, CMS-TOTEM Physics Technical Design Report, under preparation, CERN (2006).

V.A. Khoze, A.D. Martin, R. Orava and M.G. Ryskin, Eur. Phys. J. C19 (2001) 313.

V.A. Khoze, A.D. Martin, and M.G. Ryskin, Eur. Phys. J. C181 (2000) 167; Nucl. Phys. B (Proc. Suppl.) 99 (2001) 213; V.A. Khoze, A.D. Martin and M.G. Ryskin, hep-ph / 0609312 ; K. G. Boreskov, A.B. Kaidalov, V.A. Khoze, A.D. Martin and M.G. Ryskin, hep-ph / 0506211 and references therein.

The Helsinki Group (J. Kalliopuska, J.W. Lämsä, T. Mäki, N. Marola, R. Orava, K. Österberg, M. Ottela and S. Tapprogge), TOTEM forward measurements: exclusive central diffraction, in Proceedings of the Workshop on the implications of HERA for LHC physics, CERN-2005-014, 14 December 2005, p. 448.

L. Frankfurt, M. Strikman, Ch. Weiss, hep-ph / 0507286 . The dipole picture was originally introduced by A. Mueller, Nucl. Phys. B415 (1994) 373.

V.A. Khoze, A.D. Martin and M.G. Ryskin, hep-ph/0006005, In Proceedings of $8^{\text {th }}$ Int. Workshop on Deep Inelastic Scattering and QCD (DIS2000), World Scientific, 2001, p. 592; Eur. Phys. J. C19 (2001) 477; Erratum: ibid. C20 (2001) 599.

\footnotetext{
${ }^{11}$ The acceptance in Bjorken-x could be significantly increased by installing additional detectors at $19 \mathrm{~m}$ and/or at $60-140$ meters from the interaction point (Jerry Lämsä and Risto Orava, CERN, 2006).
} 
F.E. Close and G.A. Schuler, Phys. Lett. B464 (1999) 279; WA102 Collaboration, hepph/9908253; Phys. Lett. B467 (1999) 165.

N. Kochelev, hep-ph/9902203; N.I. Kochelev, T. Morii, A.V. Vinnikov, Phys. Lett. B457 (1999) 202; A. Kirk, O. Villalobos Baillie, hep-ph/9811230.

M.G. Albrow and A. Rostovtsev, Fermilab-PUB-00173 (2000).

The Helsinki Group (J. Kalliopuska, J. Lämsä, T. Mäki, N. Marola, R. Orava, K. Österberg, M. Ottela), HIP-2003-11/EXP (2003); DeRoeck, V.A. Khoze, A.D. Martin, R. Orava and M.G. Ryskin, Eur. Phys. J. C25 (2002) 391; see also: The Helsinki group (R. Orava), Forward Physics at the LHC, An invited talk at the 4th Nordic LHC Physics Workshop, Stockholm 22-24 November 2001, R. Orava, Diffraction at the LHC, Workshop on Diffractive Physics, LISHEP 2002, Rio de Janeiro, Brazil, 4-6 February 2002, (the acceptance and resolution figures refer to the simulation studies by J. Lämsä and R. Orava.)

V.A. Khoze, A.D. Martin and M.G. Ryskin, Eur. Phys. J. C23 (2002) 311.

V.A. Khoze, A.D. Martin and M.G. Ryskin, Phys. Lett. B401 (1997) 30.

A.B. Kaidalov, V.A. Khoze, A.D. Martin and M.G. Ryskin, Eur. Phys. J. C33 (2004) 387.

A.B. Kaidalov, V.A. Khoze, A.D. Martin and M.G. Ryskin, Eur. Phys. J. C33 (2004) 261.

J. Monk and A. Pilkington, Comput. Phys. Commun. 175 (2006) 232.

V.A. Khoze, A.D. Martin and M.G. Ryskin, Phys.Lett. B401 (1997) 30. 